ketonuria. Treatment with carnitine and biotin was followed by clinical improvement and normal urine organic acids. One patient in the early onset group of the London study was treated with biotin and lived to 6 years of age. The outcome for patients with propionic acidemia is generally poor, although there is a wide variation.

Propionic acidemia (ketotic hyperglycinemia) should be distinguished from non-ketotic hyperglycinemia which is characterized by hypotonia, lethargy and seizures beginning on the first day of life. Some infants succumb within a few weeks, whereas others develop mental retardation and extrapyramidal signs (Menkes JH. Textbook of Child Neurology 3rd Ed., Philadelphia, Lea \& Febiger 1981).

\title{
LEIGH ENCEPHALOPATHY
}

Histologic and biochemical analyses of muscle biopsies from 33 patients with Leigh encephalopathy were performed at the National Institute of Neuroscience, Tokyo and Tokushima University School of Medicine, Japan. Cytochrome c oxidase activity was decreased or absent in 7 patients (21\%), 10 patients $(30 \%)$ had biochemical defects including 2 with pyruvate dehydrogenase complex, 4 with cytochrome c oxidase, 1 with NADHcytochrome c reductase and 3 with multiple complex deficiencies. None had DNA deletions in the muscle mitochondria. (Nagai $\mathrm{T}$ et al. Leigh encephalopathy: histologic and biochemical analyses of muscle biopsies. Pediatr Neurol Sept/Oct 1992; $\underline{8}: 328-332$.) (Communications: Dr. Nagai, Division of UItrastructural Research, National Institute of Neuroscience, NCNP, Kodaira, Tokyo 187, Japan.)

COMMENT. A mitochondrial DNA mutation in the ATPase 6 gene was reported in 7 of 40 patients with neuropathologically or MRI defined Leigh syndrome but no known biochemical defect (Santorelli FM et al. Ann Neurol Sept $1992 ; \underline{32}: 467)$. A high abundance of the "NARP" mutation (neuropathy, ataxia, and retinitis pigmentosa) can cause Leigh syndrome and should be looked for in patients without biochemical defects. In another report, a 7 year old girl presented with a partial pyruvate carboxylase deficiency and basal ganglia lesions compatible with Leigh's disease (Schrank WI et al. Ann Neurol Sept $1992 ; 32: 468)$. The biochemical defects in Leigh encephalopathy are probably heterogeneous.

\section{NEUROPATHIES}

\section{HMSN I: EARLY DIAGNOSIS}

The value of nerve conduction velocities in the detection of hereditary motor sensory neuropathy type I (HMSN I) in children at risk was determined in 36 children under 10 years of age at the University of Western Ontario, London, Ontario, Canada. Clinical signs and slowed motor nerve conduction velocities were found in 17 of the 36 children who had 1 parent with 